\title{
Categorical Mirror Symmetry: The Elliptic Curve
}

\author{
Alexander Polishchuk ${ }^{\dagger}$ and Eric Zaslow ${ }^{\dagger \dagger}$ \\ Department of Mathematics, Harvard University, Cambridge, MA 02138, USA
}

\begin{abstract}
We describe an isomorphism of categories conjectured by Kontsevich. If $M$ and $\widetilde{M}$ are mirror pairs then the conjectural equivalence is between the derived category of coherent sheaves on $M$ and a suitable version of Fukaya's category of Lagrangian submanifolds on $\widetilde{M}$. We prove this equivalence when $M$ is an elliptic curve and $\widetilde{M}$ is its dual curve, exhibiting the dictionary in detail.
\end{abstract}

\footnotetext{
$\dagger$ email: apolish@math.harvard.edu
}

†† email: zaslow@math.harvard.edu 


\section{Introduction and Summary}

Mirror symmetry - equivalence between superconformal sigma models on certain pairs of Calabi-Yau spaces - has grown into an industry since its discovery in 1989 [1] and the subsequent translation into enumerative geometry in [2]. Nevertheless, its origins remain mysterious. In 1994, Kontsevich conjectured that mirror symmetry could be interpreted as an equivalence of categories over mirror pairs of spaces [3]. While this would not explain mirror symmetry, it may be a way of putting it on more algebraic grounds. As it currently stands - relating periods of Picard-Fuchs solutions in an appropriate basis to GromovWitten invariants [4] - mirror symmetry is rather difficult to define rigorously. Further, it has been pointed out in [5] that the appropriate moduli space for string theory should be more than just the Kähler and complex moduli of the Calabi-Yau manifold. Kontsevich introduced the constructions considered here in part as a way of naturally extending this moduli space (see also [6]).

All of the above work had been done without the knowledge of D-branes. On a CalabiYau three-fold (in Type IIB), D-branes correspond to minimal Lagrangian submanifolds with unitary local systems (by which we mean flat $U(n)$ gauge bundles). The conjecture of [7] states that these objects are responsible for mirror symmetry. For tori, K3, and certain orbifolds of tori, there is evidence for such a description [8], [9], [10], [11]. We find it appealing, then, that Kontsevich's conjecture nicely incorporates these objects on the mirror side. Indeed, the conjecture of [7] may now be reinterpreted as a consequence of the correspondence between distinguished objects in two equivalent categories.

Kontsevich's conjecture is that if $M$ and $\widetilde{M}$ are mirror pairs then $\mathcal{D}^{b}(M)$, the bounded derived category of coherent sheaves on $M$, is equivalent to the derived category of a suitable enlargement of the category $\mathcal{F}(\widetilde{M})$ of minimal Lagrangian submanifolds on $\widetilde{M}$ with unitary local systems: $\mathcal{D}^{b}(M) \cong \mathcal{D}^{b}(\mathcal{F}(\widetilde{M})) \cdot \mathcal{F}(\widetilde{M})$ was introduced by Fukaya in [12] and refined by Kontsevich [3] for the conjecture. $\mathcal{D}^{b}(\mathcal{F}(\widetilde{M}))$ denotes the derived category of the $A^{\infty}$-category $\mathcal{F}(\widetilde{M})$, constructed in [3] using twisted complexes. However, in the case of elliptic curves one doesn't need such a construction. Instead, we form a category $\mathcal{F}^{0}(\widetilde{M})$ from $\mathcal{F}(\widetilde{M})$ and provel

$$
\mathcal{D}^{b}(M) \cong \mathcal{F}^{0}(\widetilde{M})
$$

for the elliptic curve. In general, it seems that the category $\mathcal{D}^{b}(\mathcal{F}(\widetilde{M}))$ has to be further localized to have a chance at equivalence with $\mathcal{D}^{b}(M)$.

${ }^{1}$ In the notation of [3], $\mathcal{F}^{0}(\widetilde{M})$ would be called $H(\mathcal{F}(\tilde{M}))$. 
The torus is the first non-trivial check of this conjecture [3]. Here $M=E_{\tau}$ is an elliptic curve with modular parameter $\tau$ and $\widetilde{M}=\widetilde{E}^{\rho}$ is a torus (the complex structure is irrelevant here) with complexified Kähler parameter $\rho=i A+b$, where $A$ is the area and $b$ defines a class in $H^{2}(\widetilde{M} ; \mathbf{R}) / H^{2}(\widetilde{M} ; \mathbf{Z})$. The mirror map is

$$
\rho \leftrightarrow \tau
$$

(due to the positivity of $A$ and the periodicity of $b$, the more natural parameter is perhaps $q=\exp (2 \pi i \rho))$. The equivalence that we detail in this simple case is non-trivial and involves relations among theta functions and sections of higher rank bundles, as well. Note that our equivalence provides some $A^{\infty}$-extension of the derived category of coherent sheaves on an elliptic curve which we are unable to describe in a natural way.

In the next section, we describe the derived category of coherent sheaves on a manifold, and then specifically on an elliptic curve. Unfortunately, the elliptic curve is the only Calabi-Yau for which $\mathcal{D}^{b}$ is so well understood (the case of K3 is discussed in [13]). In section three, we discuss $\mathcal{F}$, Kontsevich's generalization of Fukaya's category; the category $\mathcal{F}^{0}$; and its definition for elliptic curves. In section four we address the equivalence

$$
\mathcal{D}^{b}\left(E_{\tau}\right) \cong \mathcal{F}^{0}\left(\widetilde{E}^{\tau}\right)
$$

informally, in the simplest case, giving explicit examples along the way. The general proof of (1.1) is our Main Theorem, and comprises section five. Concluding remarks and speculations may be found in section six. A table of contents is listed below. A physicist interested in getting a gist for the equivalence may wish to begin with the simple example of section four. 


\section{Introduction}

\section{The Derived Category of Coherent Sheaves}

2.1 Coherent Sheaves

2.2 The Derived Category

2.3 Vector Bundles on an Elliptic Curve

3. Fukaya's Category, $\mathcal{F}(\widetilde{M})$

3.1 Definition

$3.2 A^{\infty}$ Structure

$3.3 \mathcal{F}^{0}(\widetilde{M})$

\section{The Simplest Example}

\section{Proof of Categorical Equivalence}

5.1 The Functor $\Phi$

$5.2 \Phi \circ m_{2}=m_{2} \circ \Phi$

\subsection{Isogeny}

\subsection{The General Case}

\subsection{Extension to Torsion Sheaves}

\section{Conclusions}

\section{The Derived Category of Coherent Sheaves}

\subsection{Coherent Sheaves}

Holomorphic vector bundles are locally free sheaves, by which we mean that the space of sections over a neighborhood $U \ni z$ is $\mathcal{O} \oplus \ldots \oplus \mathcal{O}=\mathcal{O}^{\oplus r}$, where $r$ is the rank of the vector bundle. Here $\mathcal{O}$ is the sheaf of holomorphic functions on $U$. A coherent sheaf $\mathcal{S}$ admits a local presentation as an exact sequence $\mathcal{O}^{\oplus p} \rightarrow \mathcal{O}^{\oplus q} \rightarrow \mathcal{S} \rightarrow 0$, and as a result a syzygy (the local version of projective resolution), i.e. an exact sequence of sheaves

$$
\mathcal{E}_{n} \rightarrow \mathcal{E}_{n-1} \rightarrow \ldots \rightarrow \mathcal{E}_{0} \rightarrow \mathcal{S} \rightarrow 0
$$

where the $\mathcal{E}_{i}$ are locally free. The complex $\left(\mathcal{E}\right.$.) has homology $H_{0}(\mathcal{E}$. $)=\mathcal{S}$, all others zero by exactness. Therefore, we can think of $\mathcal{S}$ as roughly equivalent to a complex of 
locally free sheaves, if we only take homology. An example of a non-locally free sheaf is the skyscraper sheaf $\mathcal{O}_{z_{0}}$ over a point on a one-dimensional manifold, given by the exactness of the following (local) sequence:

$$
\mathcal{O} \stackrel{z-z_{0}}{\longrightarrow} \mathcal{O} \longrightarrow \mathcal{O}_{z_{0}} \longrightarrow 0 .
$$

The map $\left(z-z_{0}\right)$ means multiplication by $z-z_{0}$. Expanding functions in a power series around $z_{0}$, only the constants are not in the image of this map. Around every other point we can find a neighborhood such that the map is surjective, and so we say that the stalk over $z_{0}$ is non-trivial, but trivial over every other point. The rank of the sheaf $\mathcal{O}_{z_{0}}$ is thus zero, but its degree is one, since there is a global section given by a choice of constant over $z_{0}$. The kernel of the map $z-z_{0}$ is the sheaf of holomorphic functions vanishing at $z_{0}$. More generally, replacing multiplication by $\left(z-z_{0}\right)$ above by multiplication by $\left(z-z_{0}\right)^{n}$, we get the definition of the sheaf $\mathcal{O}_{n z_{0}}$ supported at the point $z_{0}$. Every coherent sheaf on a complex curve is a direct sum of vector bundles and these "thickened" skyscraper sheaves. The reason for this is that for any coherent sheaf, we can form the torsion part $\mathcal{F}_{\text {tor }}$, which is a direct sum of thickened skyscrapers. $\mathcal{F}_{\text {tor }}$ fits into the exact sequence

$$
0 \longrightarrow \mathcal{F}_{\text {tor }} \longrightarrow \mathcal{F} \longrightarrow \mathcal{G} \longrightarrow 0
$$

where $\mathcal{G}=\mathcal{F} / \mathcal{F}_{\text {tor }}$ is locally free (a vector bundle). However, one finds no nontrivial extensions by $\mathcal{F}_{\text {tor }}$, and so $\mathcal{F}=\mathcal{G} \oplus \mathcal{F}_{\text {tor }}$ as claimed.

\subsection{The Derived Category}

The (bounded) derived category $\mathcal{D}^{b}(M)$ of coherent sheaves on $M$ is obtained from the category of (bounded) complexes of coherent sheaves by inverting quasi-isomorphisms - i.e., morphisms inducing isomorphisms on all cohomology sheaves of the complex. In the case when $M$ is a complex projective curve every object of $\mathcal{D}^{b}(M)$ is isomorphic to the direct sum of objects of the form $F[n]$ where $F$ is a coherent sheaf on $M, F[n]$ denotes the complex with the only non-zero term (equal to $F$ ) in degree $-n$. Thus, every object of $\mathcal{D}^{b}(M)$ is a direct sum of objects of the form $F[n]$ where $F$ is either a vector bundle or has support at a point. 


\subsection{Vector Bundles on an Elliptic Curve}

We collect here some facts about theta functions, concentrating on the elliptic curve [14]. Line bundles on an elliptic curve $E=\mathbf{C} / \Lambda$ have a simple description. The lattice $\Lambda$ has 2 generators, one of which (by a rescaling) can be chosen to be $z \rightarrow z+1$. Then $X=\mathbf{C}^{*} / \mathbf{Z}$, where the coordinate on $\mathbf{C}^{*}$ is $u=\exp (2 \pi i z)$, and the action of the lattice is $u \rightarrow q u$, where $q=\exp (2 \pi i \tau)$. Call $\pi^{\prime}$ the projection map $\pi^{\prime}: \mathbf{C}^{*} \rightarrow E$. Now using the fact that $H^{1}\left(\mathbf{C}^{*}, \mathcal{O}\right)=H^{2}\left(\mathbf{C}^{*}, \mathcal{O}\right)=0$, the long exact sequence associated to the exact

sheaf sequence $0 \rightarrow \mathbf{Z} \rightarrow \mathcal{O} \rightarrow \mathcal{O}^{*} \rightarrow 0$ tells us that $H^{1}\left(\mathbf{C}^{*}, \mathcal{O}^{*}\right) \cong H^{2}\left(\mathbf{C}^{*}, \mathbf{Z}\right)$. This map is by definition the first Chern class, and therefore line bundles on $\mathbf{C}^{*}$ are determined by the first Chern class. But since there are no non-trivial two-forms on $\mathbf{C}^{*}$, we learn that the pull-back of any line bundle $L$ over $E$ is trivial over $\mathbf{C}^{*}$.

More generally, the pull-back of every vector bundle on $E_{q}$ to $\mathbf{C}^{*}$ is trivial since all vector bundles on $E$ are obtained from line bundles by successive extensions. Thus, all vector bundles on $E$ are obtained by the following construction. Let $V$ be an $r$-dimensional vector space and $A: \mathbf{C}^{*} \rightarrow G L(V)$ a holomorphic, vector-valued function. We define the rank $r$ holomorphic vector bundle $F_{q}(V, A)$ on $E$ by taking the quotient

$$
F_{q}(V, A)=\mathbf{C}^{*} \times V /(u, v) \sim(u q, A(u) \cdot v) .
$$

The theory of vector bundles on elliptic curves can be rephrased as a classification of such holomorphic functions up to equivalence of $A(u)$ and $B(q u) A(u) B(u)^{-1}$ where $B: \mathbf{C}^{*} \rightarrow$ $\operatorname{GL}(V)$.

When $V=\mathbf{C}$ and $A=\varphi$ a holomorphic function, we call $L_{q}(\varphi)$ the line bundle constructed in this way (or simply $L(\varphi)$ if there is no ambiguity). We define $L \equiv L_{q}\left(\varphi_{0}\right)$, where $\varphi_{0}(u)=\exp (-\pi i \tau-2 \pi i z)=q^{-1 / 2} u^{-1}$. The line bundle $L:=L\left(\phi_{0}\right)$ will play a distinguished role in our considerations since the classical theta function is a section of $L$. Every holomorphic line bundle on $E_{q}$ has form $t_{x}^{*} L \otimes L^{n-1}$ for some $n \in \mathbf{Z}$ and $x \in E_{q}$, where $t_{x}$ is the map of translation by $x$ on $E_{q}$. For a torus, the theta function has as indices $\tau$, the modular parameter, and $\left(c^{\prime}, c^{\prime \prime}\right) \in \mathbf{R}^{2} / \mathbf{Z}^{2}$, a translation parametrizing different line bundles of the same degree. The theta function is defined to be

$$
\theta\left[c^{\prime}, c^{\prime \prime}\right](\tau, z)=\sum_{m \in \mathbf{Z}} \exp \left\{2 \pi i\left[\tau\left(m+c^{\prime}\right)^{2} / 2+\left(m+c^{\prime}\right)\left(z+c^{\prime \prime}\right)\right]\right\} .
$$

If $\left(c^{\prime}, c^{\prime \prime}\right)=(0,0)$, we will simply use the notation $\theta(\tau, z)$. Then the $n$ functions $\theta[a / n, 0](n \tau, n z), a \in \mathbf{Z} / n \mathbf{Z}$, are the global sections of $L^{n}$. 
Now consider the natural $r$-fold covering $\pi_{r}: E_{q^{r}} \rightarrow E_{q}$ which sends $u$ to $u$. Clearly, $\pi_{r}^{-1}(u)=\left\{u, u q, u q^{2}, \ldots, u q^{r-1}\right\}$. We have the natural functors of pull-back and push-forward associated with $\pi_{r}$. More concretely, $\pi_{r}^{*} F_{q}(V, A)=F_{q^{r}}\left(V, A^{r}\right)$, while $\pi_{r *} F_{q}(V, A)=F_{q}\left(V \otimes \mathbf{C}^{r}, \pi_{r *} A\right)$, where $\pi_{r *} A\left(v \otimes e_{i}\right)=v \otimes e_{i+1}$ for $i=1, \ldots, r-1$, $\pi_{r *} A\left(v \otimes e_{r}\right)=A v \otimes e_{1}$. It is easy to see that the functor of pull-back commutes with tensor product and duality. Also one has natural isomorphisms

$$
\begin{gathered}
\pi_{r *}\left(F_{1} \otimes \pi_{r}^{*} F_{2}\right) \cong \pi_{r *}\left(F_{1}\right) \otimes F_{2}, \\
\left(\pi_{r *}(F)\right)^{*} \cong \pi_{r *}\left(F^{*}\right),
\end{gathered}
$$

and

$$
H^{0}\left(E_{q}, \pi_{r *}(F)\right) \cong H^{0}\left(E_{q^{r}}, F\right) .
$$

It follows that

$$
\begin{aligned}
& \operatorname{Hom}\left(F_{1}, \pi_{r *} F_{2}\right) \cong \operatorname{Hom}\left(\pi_{r}^{*} F_{1}, F_{2}\right) \\
& \operatorname{Hom}\left(\pi_{r *} F_{1}, F_{2}\right) \cong \operatorname{Hom}\left(F_{1}, \pi_{r}^{*} F_{2}\right)
\end{aligned}
$$

The following results will be useful in the sequel.

\section{Proposition 1}

Every indecomposable bundle of on $E_{q}$ is isomorphic to a bundle of the form $\pi_{r *}\left(L_{q^{r}}(\phi) \otimes F_{q^{r}}\left(\mathbf{C}^{k}, \exp N\right)\right)$, where $N$ is a constant indecomposable nilpotent matrix,

$\phi=t_{x}^{*} \varphi_{0} \cdot \varphi_{0}^{n-1}$ for some $n \in \mathbf{Z}$ and $x \in \mathbf{C}^{*}$, and $t_{x}$ represents translation by $x$.

This proposition can be deduced easily from the classification of holomorphic bundles on elliptic curves due to M. Atiyah [15].

\section{Proposition 2}

Let $\varphi=t_{x}^{*} \varphi_{0} \cdot \varphi_{0}^{n-1}$, with $n>0$. Then for any nilpotent endomorphism $N \in \operatorname{End}(V)$, there is a canonical isomorphism

$$
\mathcal{V}_{\varphi, N}: H^{0}(L(\varphi)) \otimes V \rightarrow H^{0}(L(\varphi) \otimes F(V, \exp N))
$$

Proof: $\operatorname{Put} \mathcal{V}_{\varphi, N}(f \otimes v)=\exp (D N / n) f \cdot v$, where $D=-u \frac{d}{d u}=-\frac{1}{2 \pi i} \frac{d}{d z}$. Now since $f$ is a section of $L$ we have $f(q u)=\varphi f(u)$. Now a section $w$ of $L \otimes F(V, N)$ is equivalent to a holomorphic vector-valued function of $u$ obeying $w(q u)=\varphi(u) \exp (N) \cdot w(u)$. First 
note that $D$ commutes with the operation $u \rightarrow u q$, so $[D f](u q)=D(f(u q))$. We also have $D(\varphi f)=\varphi(D+n) f$, which is easily checked. As a result, we find

$$
\begin{aligned}
\exp (D N / n) f(q u) v & =\exp (D N / n)[\varphi f(u) v] \\
& =\sum_{k=0}^{\infty} \frac{1}{k ! n^{k}} D^{k}(\varphi f) N^{k} v \\
& =\sum_{k=0}^{\infty} \frac{1}{k ! n^{k}} \varphi(D+n)^{k} f N^{k} v \\
& =\exp [(D+n) N / n] f v \\
& =\exp (N)[\exp (D N) \cdot f v]
\end{aligned}
$$

and the proposition is shown.

\section{Proposition 3}

Let $\varphi_{1}=t_{x_{1}}^{*} \varphi_{0} \cdot \varphi_{0}^{n_{1}-1}, \varphi_{2}=t_{x_{2}}^{*} \varphi_{0} \cdot \varphi_{0}^{n_{2}-1}$, and let $N_{i} \in \operatorname{End}\left(V_{i}\right), i=1,2$, be nilpotent endomorphisms. Then

$$
\begin{aligned}
& \mathcal{V}_{\varphi_{1}, N_{1}}\left(f_{1} \otimes v_{1}\right) \circ \mathcal{V}_{\varphi_{2}, N_{2}}\left(f_{2} \otimes v_{2}\right)= \\
& \mathcal{V}_{\varphi_{1} \varphi_{2}, N_{1}+N_{2}}\left[\exp \left(\frac{n_{2} N_{1}-n_{1} N_{2}}{n_{1}+n_{2}} \frac{D}{n_{1}}\right)\left(f_{1}\right) \exp \left(\frac{n_{1} N_{2}-n_{2} N_{1}}{n_{1}+n_{2}} \frac{D}{n_{2}}\right)\left(f_{2}\right)\left(v_{1} \otimes v_{2}\right)\right]
\end{aligned}
$$

where $N_{1}, N_{2}$ denote $N_{1} \otimes 1$ and $1 \otimes N_{2}$ respectively, on the right hand side, and $\circ$ denotes the natural composition of sections

$$
\begin{aligned}
H^{0}\left(L\left(\varphi_{1}\right) \otimes F\left(V_{1}, \exp N_{1}\right)\right) \otimes H^{0}\left(L\left(\varphi_{2}\right) \otimes F\left(V_{2}, \exp N_{2}\right)\right) \rightarrow \\
H^{0}\left(L\left(\varphi_{1} \varphi_{2}\right) \otimes F\left(V_{1} \otimes V_{2}, \exp \left(N_{1} \otimes 1+1 \otimes N_{2}\right)\right)\right) .
\end{aligned}
$$

Recalling that $\exp \frac{d}{d z}$ is the generator of translations, we may write formally $\exp (N$. $\left.\frac{d}{d z}\right) f(z)=f(z+N)$. In this notation, the above formula looks like

$$
\begin{aligned}
\mathcal{V}\left(f_{1} \otimes v_{1}\right) \circ \mathcal{V}\left(f_{2} \otimes v_{2}\right) & =\mathcal{V}\left(f_{1}\left(z+\frac{n_{1} N_{2}-n_{2} N_{1}}{2 \pi i n_{1}\left(n_{1}+n_{2}\right)}\right) f_{2}\left(z+\frac{n_{2} N_{1}-n_{1} N_{2}}{2 \pi i n_{2}\left(n_{1}+n_{2}\right)}\right)\left(v_{1} \otimes v_{2}\right)\right) \\
& =\mathcal{V}\left(f_{1}\left(u e^{\frac{n_{1} N_{2}-n_{2} N_{1}}{n_{1}\left(n_{1}+n_{2}\right)}}\right) f_{2}\left(u e^{\frac{n_{2} N_{1}-n_{1} N_{2}}{n_{2}\left(n_{1}+n_{2}\right)}}\right)\left(v_{1} \otimes v_{2}\right)\right) .
\end{aligned}
$$

The proof is straightforward. 


\section{Fukaya's Category, $\mathcal{F}(\widetilde{\mathcal{M}})$}

\subsection{Definition}

Let $\widetilde{M}$ be a Calabi-Yau manifold with its unique, Ricci-flat Kähler metric and Kähler form $k$ (not just the cohomology class, but the differential form). Our main example will be when $\widetilde{M}$ is a torus, $\widetilde{E}$, with flat metric $d s^{2}=A\left(d x^{2}+d y^{2}\right)$, so that its volume is equal to $A$. We also specify an element $b$ in $H^{2}(\widetilde{E} ; \mathbf{R}) / H^{2}(\widetilde{E} ; \mathbf{Z})$. We define a complexified Kähler form $\omega \equiv b+i k$.

A category is given by a set of objects and composable morphisms between objects. $A^{\infty}$ categories have additional structures on the morphisms, which we will discuss later in this section.

Objects: The objects of $\mathcal{F}(\widetilde{\mathcal{M}})$ are special Lagrangian submanifolds of $\widetilde{M}$ - i.e. minimal Lagrangian submanifolds - endowed with flat bundles with monodromies having eigenvalues of unit modulus?, and one additional structure we will discuss momentarily. We recall that a Lagrangian submanifold is one on which $k$ restricts to zero. Though Fukaya and Kontsevich have taken the submanifolds to simply be Lagrangian, we will need the minimality condition as well. 3 This, plus Kontsevich's addition of the local system, is also what one expects based on relations with D-branes [17]. Thus, an object $\mathcal{U}_{i}$ is a pair:

$$
\mathcal{U}_{i}=\left(\mathcal{L}_{i}, \mathcal{E}_{i}\right)
$$

In our example of a torus, the minimal submanifolds are just minimal lines, or geodesics (the Lagrangian property is trivially true for one-dimensional submanifolds). To define a closed submanifold in $\mathbf{R}^{2} /(\mathbf{Z} \oplus \mathbf{Z})$ the slope of the line must be rational, so can be given by a pair of integers $(p, q)$. There is another real datum needed, which is the point of interception with the line $x=0$ (or $y=0$ if $p=0$ ). In the easiest case, the rank of the unitary system is one, so that we can specify a flat line bundle on the circle by simply specifying the monodromy around the circle, i.e. a complex phase $\exp (2 \pi i \beta), \beta \in \mathbf{R} / \mathbf{Z}$.

2 Kontsevich only considered unitary local systems, or flat $U(n)$ bundles. We prove an equivalence of categories involving a larger class of objects. The Jordan blocks will be related to non-stable vector bundles over the torus. See section five for details.

3 A Lagrangian minimal (or "special Lagrangian") submanifold $L$ is a kind of calibrated submanifold [16] associated to the Calabi-Yau form, $\Omega$. This means that for a suitable complex phase of $\Omega$, we have $\left.\operatorname{Re}(\Omega)\right|_{L}=\operatorname{Vol}_{L}$, where the volume form is determined by the induced metric. Equivalently, one has $\left.k\right|_{L}=0$ and $\left.\operatorname{Im}(\Omega)\right|_{L}=0$. 
For a general local system of rank $r$ we can take $(p, q)$ to have greatest common divisor equal to $r$.

The additional structure we need is the following. A Lagrangian submanifold $L$ of real dimension $n$ in a complex $n$-fold, $\widetilde{M}$, defines not only a map from $L$ to $\widetilde{M}$ but also the Gauss map from $L$ to $V$, where $V$ fibers over $\widetilde{M}$ with fiber at $x$ equal to the space of Lagrangian planes at $T_{x} \widetilde{M}$. The space of Lagrangian planes has fundamental group equal to $\mathbf{Z}$, and we take as objects special Lagrangian submanifolds together with lfts of the Gauss map into the fiber bundle over $\widetilde{M}$ with fiber equal to the universal cover of the space of Lagrangian planes.

For our objects, we thus require more than the slope, which can be thought of as a complex phase with rational tangency, and therefore as

$$
\exp i \pi \alpha .
$$

We need a choice of $\alpha$ itself. Clearly, the $\mathbf{Z}$-degeneracy represents the deck transformations of the universal cover of the space of slopes. Shifts by integers correspond to shifts by grading of the bounded complexes in the derived category. There is no natural choice of zero in $\mathbf{Z}$.

Morphisms: The morphisms $\operatorname{Hom}\left(\mathcal{U}_{i}, \mathcal{U}_{j}\right)$ are defined as

$$
\operatorname{Hom}\left(\mathcal{U}_{i}, \mathcal{U}_{j}\right)=\mathbf{C}^{\#\left\{\mathcal{L}_{i} \cap \mathcal{L}_{j}\right\}} \otimes \operatorname{Hom}\left(\mathcal{E}_{i}, \mathcal{E}_{j}\right)
$$

where the second "Hom" in the above represents homomorphisms of vector spaces underlying the local systems at the points of intersection. There is a $\mathbf{Z}$-grading on the morphisms. If $p$ is a point in $\mathcal{L}_{i} \cap \mathcal{L}_{j}$ then it has a Maslov index $\mu(p) \in \mathbf{Z}$.

For our example, let us consider $\operatorname{Hom}\left(\mathcal{U}_{i}, \mathcal{U}_{j}\right)$, where the unitary systems have rank one, and where the lines $\mathcal{L}_{i}$ and $\mathcal{L}_{j}$ go through the origin. Then $\tan \alpha_{i}=q / p$ and $\tan \alpha_{j}=s / r$, with $(p, q)$ and $(r, s)$ both relatively prime pairs. For simplicity, one can think of the lines as the infinite set of parallel lines on the universal cover of the torus, $\mathbf{R} \oplus \mathbf{R}$. It is then easy to see that there are

$$
|p s-q r|
$$

non-equivalent points of intersection. $\operatorname{Since} \operatorname{Hom}(\mathbf{C}, \mathbf{C})$ is one dimensional, the monodromy is specified by a single complex phase $T_{i}$ at each point of intersection. For rank $n$ local systems, $T_{i}$ would be represented by an $n \times n$ matrix.

4 A discussion of the Maslov index may be found in chapter four of the first reference in [12]. 
The $\mathbf{Z}$-grading on $\operatorname{Hom}\left(\mathcal{U}_{i}, \mathcal{U}_{j}\right)$ is constant for all points of intersection in our example (they are all related by translation). If $\alpha_{i}, \alpha_{j}$ are the real numbers representing the logarithms of the slopes, as above, then for $p \in \mathcal{L}_{i} \cap \mathcal{L}_{j}$ the grading is given by

$$
\mu(p)=-\left[\alpha_{j}-\alpha_{i}\right]
$$

where the brackets represent the greatest integer. Note that $-[x]-[-x]=1$, which the Maslov index must obey for a one-fold. The Maslov index is non-symmetric. For $p \in \mathcal{L}_{i} \cap \mathcal{L}_{j}$ in an $n$-fold, $\mu(p)_{i j}+\mu(p)_{j i}=n$. The asymmetry is reassuring, as we know that $\operatorname{Hom}\left(E_{i}, E_{j}\right)$ is not symmetric in the case of bundles. It is the extra data of the lift of the Lagrangian plane which allowed us to define the Maslov index in this way.

Generally speaking, a category has composable morphisms which satisfy associativity conditions. This is not generally true for the category $\mathcal{F}(\widetilde{\mathcal{M}})$. However, we have instead on $\mathcal{F}(\widetilde{\mathcal{M}})$ an additional interesting structure making $\mathcal{F}(\widetilde{\mathcal{M}})$ an $A^{\infty}$ category. Associativity will hold cohomologically, in a way. The equivalence of categories that we will prove will involve a true category $\mathcal{F}^{0}(\widetilde{M})$, which we will construct from $\mathcal{F}(\widetilde{\mathcal{M}})$ in section 3.3.

\section{2. $A^{\infty}$ Structure}

The category $\mathcal{F}(\widetilde{\mathcal{M}})$ has an $A^{\infty}$ structure, by which we mean the composable morphisms satisfy conditions analogous to those of an $A^{\infty}$ algebra [18]. An $A^{\infty}$ algebra is a generalization of a differential, graded algebra. Namely, it is a $\mathbf{Z}$-graded vector space, with a degree one map, $m_{1}$, which squares to zero $\left(\left(m_{1}\right)^{2}=0\right)$. There are higher maps, $m_{k}: A^{\otimes k} \rightarrow A$, as well.

Definition: An $A^{\infty}$ category, $\mathcal{F}$ consists of

- A class of objects $\mathrm{Ob}(\mathcal{F})$;

- For any two objects, $X, Y$, a $\mathbf{Z}$-graded abelian group of morphisms $\operatorname{Hom}(X, Y)$;

- Composition maps

$$
m_{k}: \operatorname{Hom}\left(X_{1}, X_{2}\right) \otimes \operatorname{Hom}\left(X_{2}, X_{3}\right) \otimes \ldots \operatorname{Hom}\left(X_{k}, X_{k+1}\right) \rightarrow \operatorname{Hom}\left(X_{1}, X_{k+1}\right),
$$

$k \geq 1$, of degree $2-k$, satisfying the condition

$$
\sum_{r=1}^{n} \sum_{s=1}^{n-r+1}(-1)^{\varepsilon} m_{n-r+1}\left(a_{1} \otimes \ldots \otimes a_{s-1} \otimes m_{r}\left(a_{s} \otimes \ldots \otimes a_{s+r-1}\right) \otimes a_{s+r} \otimes \ldots \otimes a_{n}\right)=0
$$

for all $n \geq 1$, where $\varepsilon=(r+1) s+r\left(n+\sum_{j=1}^{s-1} \operatorname{deg}\left(a_{j}\right)\right)$. 
An $A^{\infty}$ category with one object is called an $A^{\infty}$ algebra. The first condition $(n=1)$ says that $m_{1}$ is a degree one operator satisfying $\left(m_{1}\right)^{2}=0$, so it is a co-boundary operator which we can denote $d$. The second condition says that $m_{2}$ is a degree zero map satisfying $d\left(m_{2}\left(a_{1} \otimes a_{2}\right)\right)=m_{2}\left(d a_{1} \otimes a_{2}\right)+(-1)^{\operatorname{deg}\left(a_{1}\right)} m_{2}\left(a_{1} \otimes d a_{2}\right)$, so $m_{2}$ is a morphism of complexes and induces a product on cohomologies. The third condition says that $m_{2}$ is associative at the level of cohomologies.

The $A^{\infty}$ structure on Fukaya's category is given by summing over holomorphic maps (up to projective equivalence) from the disc $D^{2}$, which take the components of the boundary $S^{1}=\partial D^{2}$ to the special Lagrangian objects. An element $u_{j}$ of $\operatorname{Hom}\left(\mathcal{U}_{j}, \mathcal{U}_{j+1}\right)$ is represented by a pair

$$
u_{j}=t_{j} \cdot a_{j}
$$

where $a_{j} \in \mathcal{L}_{j} \cap \mathcal{L}_{j+1}$, and $t_{j}$ is a matrix in $\operatorname{Hom}\left(\left.\mathcal{E}_{j}\right|_{a_{j}},\left.\mathcal{E}_{j+1}\right|_{a_{j}}\right)$.

$$
m_{k}\left(u_{1} \otimes \ldots \otimes u_{k}\right)=\sum_{a_{k+1} \in \mathcal{L}_{1} \cap \mathcal{L}_{k+1}} C\left(u_{1}, \ldots, u_{k}, a_{k+1}\right) \cdot a_{k+1},
$$

where (notation explained below)

$$
C\left(u_{1}, \ldots, u_{k}, a_{k+1}\right)=\sum_{\phi} \pm \mathrm{e}^{2 \pi i \int \phi^{*} \omega} \cdot P \mathrm{e}^{\oint \phi^{*} \beta},
$$

is a matrix in $\operatorname{Hom}\left(\left.\mathcal{E}_{1}\right|_{a_{k+1}},\left.\mathcal{E}_{k+1}\right|_{a_{k+1}}\right)$. Here we sum over (anti-)holomorphic maps $\phi$ : $D^{2} \rightarrow \widetilde{M}$, up to projective equivalence, with the following conditions along the boundary: there are $k+1$ points $p_{j}=\mathrm{e}^{2 \pi i \alpha_{j}}$ such that $\phi\left(p_{j}\right)=a_{j}$ and $\phi\left(\mathrm{e}^{2 \pi i \alpha}\right) \in \mathcal{L}_{j}$ for $\alpha \in\left(\alpha_{j-1}, \alpha_{j}\right)$. In the above, $\omega=b+i k$ is the complexified Kähler form, the sign is determined by an orientation in the space of holomorphic maps (it will always be positive for us), and $P$ represents a path-ordered integration, where $\beta$ is the connection of the flat bundle along the local system on the boundary. Note that in the case of all trivial local systems $(\beta \equiv 0)$, the weighting is just the exponentiated complexified area of the map. The path-ordered integral is defined by

$$
P \mathrm{e}^{\oint \phi^{*} \beta}=P \mathrm{e}^{\int_{\alpha_{k}}^{\alpha_{k+1}} \beta_{k} d \alpha} \cdot t_{k} \cdot P \mathrm{e}^{\int_{\alpha_{k-1}}^{\alpha^{k}} \beta_{k-1} d \alpha} \cdot t_{k-1} \cdot \ldots \cdot t_{1} \cdot P \mathrm{e}^{\int_{\alpha_{k+1}}^{\alpha_{1}} \beta_{1} d \alpha}
$$

(this formula is easily understood by reading right to left). Following the integration along the boundary, we get a homomorphism from $\mathcal{E}_{1}$ to $\mathcal{E}_{k+1}$. The path ordering symbol is a bit superfluous above, since the integral is one-dimensional, but we have retained it for exposition.

Fukaya has shown the $A^{\infty}$ structure of his category in [12]. Our modifications should not affect the proof. 
3.3. $\mathcal{F}^{0}(\widetilde{M})$

To define a true category which can be checked against the derived category, we simply take $H^{0}$ of all the morphisms (recall that they have the structure of complexes). In our case of the elliptic curve, since $m_{1}=d=0$ the cohomology complex is the same as the original complex, and so we simply take the degree zero piece of Hom - however, the construction is valid in general. Recall that $m_{2}$ was associative at the level of cohomlogies. This reduces to true associativity of the composition of morphisms. We call this category form in this way $\mathcal{F}^{0}(\widetilde{M})$. For our example $m_{2}$ has degree zero, survives this restriction and is associative. The higher $m$ 's are profected to zero in this category, so our equivalence will be defined by constructing a dictionary of objects and checking compatibility with $m_{2}$.

\section{The Simplest Example}

We now demonstrate the equivalence between the derived category of coherent sheaves on an elliptic curve and the category $\mathcal{F}^{0}(\widetilde{E})$ on the dual torus, in the simplest possible example. A complete proof will be given in the next section.

We begin with an elliptic curve $E=\mathbf{C} /(\mathbf{Z} \oplus \tau \mathbf{Z}) \cdot \tau$ defines the complex structure of $E$. Its mirror torus is $\widetilde{E}=\mathbf{R}^{2} /(\mathbf{Z} \oplus \mathbf{Z})$, with Kähler (metric) structure defined below, and related to $\tau$ by the mirror map.

Let us first construct the dictionary. Line bundles of degree (first Chern class) $d$ correspond to lines of slope $d$. With this simple rule, and the definition of the compositions in both categories, we can try to compare the map $m_{2}$ between morphisms directly. the moment we set all translations (which parametrize bundles of the same rank and degree) to zero, which means in part, on the Fukaya side (as we shall see) that all lines pass through the origin (we assume an origin has been chosen for the elliptic curve and its mirror). We also set all holonomies to be zero (trivial local systems). We will return to non-zero translations and holonomies later in this section. We define the Kähler parameter to be

$$
\rho=b+i A
$$

where $A$ is the area of the torus, and $b$ describes a two-form of the same name: $b=$ $b d x \wedge d y \in H^{2}(\widetilde{E} ; \mathbf{R}) / H^{2}(\widetilde{E} ; \mathbf{Z})$. In terms of the complexified Kähler form $\omega$ of the previous

5 Since $m_{2}$ has degree zero with respect to the $\mathbf{Z}$-grading on morphisms, we can ignore the universal cover of the space of lagrangian planes and just consider the slope of the lines. 
section, $\rho=\int_{\widetilde{E}} \omega$ (and $A=k$ ). We set $b=0$ to begin with, so $\rho=i A$. The mirror map says that $\widetilde{E}$ is dual to $E$ when $\rho=\tau$.

Let

$$
\begin{aligned}
& \mathcal{L}_{1}=(1,0), \\
& \mathcal{L}_{2}=(1,1), \\
& \mathcal{L}_{3}=(1,2) .
\end{aligned}
$$

On the derived category side, then, we are considering line bundles of degrees $0,1,2$. Note that $\mathcal{L}_{1}=\mathcal{O}$, the sheaf of holomorphic functions. We can define $L \equiv \mathcal{L}_{2}$; then $\mathcal{L}_{3}=L^{2}$, and

$$
\begin{aligned}
& \operatorname{Hom}\left(\mathcal{L}_{1}, \mathcal{L}_{2}\right)=H^{0}(L), \\
& \operatorname{Hom}\left(\mathcal{L}_{2}, \mathcal{L}_{3}\right)=H^{0}(L), \\
& \operatorname{Hom}\left(\mathcal{L}_{1}, \mathcal{L}_{3}\right)=H^{0}\left(L^{2}\right) .
\end{aligned}
$$

The product of global sections gives us a map

$$
m_{2}: H^{0}(L) \otimes H^{0}(L) \longrightarrow H^{0}\left(L^{2}\right) .
$$

The theta function $\theta(\tau, z)$ is the unique global section of $L$, and the decomposition of the product of theta functions into two sections of $L^{2}$ is known as the "addition formula."

Let's see what we get from the Lagrangian lines $\mathcal{L}_{i}$ (we use the same notation as for bundles) We have three lines through the origin of slope 0,1 , and 2 respectively. Note that $\mathcal{L}_{1} \cap \mathcal{L}_{2}=\left\{\mathrm{e}_{1}\right\}$, where the origin $\mathrm{e}_{1} \equiv(0,0)$. Also, $\mathcal{L}_{2} \cap \mathcal{L}_{3}=\left\{\mathrm{e}_{1}\right\}$, while $\mathcal{L}_{2} \cap \mathcal{L}_{3}=\left\{\mathrm{e}_{1}, \mathrm{e}_{2}\right\}$, where $\mathrm{e}_{2} \equiv(1 / 2,0)$. On the left hand side of (4.1), $\mathrm{e}_{1}$ represents the theta function $\theta(\tau, z)$.6 On the right hand side, the $\mathrm{e}_{i}$ represent a distinguished basis of the two-dimensional space of sections of $L^{2}$ (see section 2.3 for details). We define this basis correspondence by

$$
\begin{aligned}
& \mathrm{e}_{1} \leftrightarrow \theta[0,0](2 \tau, 2 z), \\
& \mathrm{e}_{2} \leftrightarrow \theta[1 / 2,0](2 \tau, 2 z) .
\end{aligned}
$$

We will use the mirror map

$$
\tau=\rho
$$

to check the correspondence of the $m_{2}$ product.

6 As we point out again in the next section, the notation is ambiguous: the section being represented by $\mathrm{e}_{1}$ depends on the two lines of which we take it as an intersection point. 
The map $m_{2}$ is:

$$
m_{2}\left(\mathrm{e}_{1} \otimes \mathrm{e}_{1}\right)=C\left(\mathrm{e}_{1}, \mathrm{e}_{1}, \mathrm{e}_{1}\right) \cdot \mathrm{e}_{1}+C\left(\mathrm{e}_{1}, \mathrm{e}_{1}, \mathrm{e}_{2}\right) \cdot \mathrm{e}_{2},
$$

where we use Fukaya's procedure (3.1) to compute the matrix elements, $C$. The holomorphic maps from the discs are specified by the triangles bounded by $\mathcal{L}_{1}, \mathcal{L}_{2}$, and $\mathcal{L}_{3}$ with vertices given by the arguments of $C$. Thus, $C\left(\mathrm{e}_{1}, \mathrm{e}_{1}, \mathrm{e}_{1}\right)$ is given by summing all triangles with the origin as vertex. Looking at the universal cover of the torus, we need (up to translation) triangles with lattice points as vertices and sides of slope 0,1 , and 2 . Consider the base of the triangle, which must be integral length, say $n$, running from $(0,0)$ to $(n, 0)$. The third vertex is $(2 n, 2 n)$, which is a lattice point. Thus all the triangles are indexed by the integers, and the $n^{\text {th }}$ triangle has area $n^{2}$ times the area $A$. To determine $C\left(\mathrm{e}_{1}, \mathrm{e}_{1}, \mathrm{e}_{2}\right)$ we use the triangles with vertices $(0,0),(0, n+1 / 2),(2 n+1,2 n+1)$ and area $A(n+1 / 2)^{2}$.

Therefore,

$$
\begin{aligned}
& C\left(\mathrm{e}_{1}, \mathrm{e}_{1}, \mathrm{e}_{1}\right)=\sum_{n=-\infty}^{\infty} \exp \left[-2 \pi A n^{2}\right] \\
& C\left(\mathrm{e}_{1}, \mathrm{e}_{1}, \mathrm{e}_{2}\right)=\sum_{n=-\infty}^{\infty} \exp \left[-2 \pi A(n+1 / 2)^{2}\right] .
\end{aligned}
$$

Note that the triangles for $n \geq 0$ and $n<0$ are related by the $\mathbf{Z}_{2}$ automorphism of the system (torus plus configuration of Lagrangian lines); nevertheless, we treat them as distinct. We recognize (see section 2.3) the coefficients $C$ as theta functions evaluated at $z=0$ (with no shifts). Specifically,

$$
\begin{aligned}
& C\left(\mathrm{e}_{1}, \mathrm{e}_{1}, \mathrm{e}_{1}\right)=\theta[0,0](i 2 A, 0)=\theta[0,0](2 \rho, 0), \\
& C\left(\mathrm{e}_{1}, \mathrm{e}_{1}, \mathrm{e}_{2}\right)=\theta[1 / 2,0](i 2 A, 0)=\theta[1 / 2,0](2 \rho, 0) .
\end{aligned}
$$

The product $m_{2}$ therefore precisely contains the information of the addition formula

$$
\theta(\rho, z) \theta(\rho, z)=\theta[0,0](2 \rho, 0) \theta[0,0](2 \rho, 2 z)+\theta[1 / 2,0](2 \rho, 0) \theta[1 / 2,0](2 \rho, 2 z) .
$$

We now consider the case where the third line doesn't pass through the point of intersection of the other two. Call $\alpha$ the (positive) $x$-intecept of the closest line of slope two, so $0<\alpha<1 / 2$. Then the points of $\mathcal{L}_{1} \cap \mathcal{L}_{3}$ are $(\alpha, 0),(1 / 2+\alpha, 0)$. If we label the $\alpha$-shifted basis $\mathrm{e}_{1, \alpha}, \mathrm{e}_{2, \alpha}$, then we have

$$
\begin{aligned}
& C\left(\mathrm{e}_{1}, \mathrm{e}_{1}, \mathrm{e}_{1, \alpha}\right)=\sum_{n=-\infty}^{\infty} \exp \left(-2 \pi A[n+\alpha)^{2}\right]=\theta[\alpha, 0](2 \rho, 0) \\
& C\left(\mathrm{e}_{1}, \mathrm{e}_{1}, \mathrm{e}_{1, \alpha}\right)=\sum_{n=-\infty}^{\infty} \exp \left[-2 \pi A(n+1 / 2+\alpha)^{2}\right]=\theta[1 / 2+\alpha, 0](2 \rho, 0) .
\end{aligned}
$$


One also needs to know the rules for identifying the sections corresonding to the shifted basis, and the obvious guess is

$$
\begin{aligned}
& \mathrm{e}_{1, \alpha} \leftrightarrow \theta[\alpha, 0](2 \tau, 2 z) \\
& \mathrm{e}_{2, \alpha} \leftrightarrow \theta[1 / 2+\alpha, 0](2 \tau, 2 z) .
\end{aligned}
$$

In fact, this is almost correct. Some phases need to be added, after which the composition becomes once again equivalent to the addition formula, now with $\alpha$-dependent shifts, as indicated. The exact formulas are given in the next section. The appearance of these phases is not unexpected, since there is a certain arbitrariness in the choice of $\tau$-dependence of the phase in the definition of the theta function.

Let us return to zero translation, but add holonomy to $\mathcal{L}_{3}$. That is, we take the flat line bundle with connection $2 \pi i \beta d t_{3}$ where $t_{3} \sim t_{3}+1$ is a coordinate along $\mathcal{L}_{3}$. The matrix elements $C$ then have to be weighted by $\exp [-2 \pi$ (Area) $+2 \pi i \oint \beta d t]$. This gives

$$
\begin{aligned}
& C\left(\mathrm{e}_{1}, \mathrm{e}_{1}, \mathrm{e}_{1}\right)=\sum_{n=-\infty}^{\infty} \exp \left[-2 \pi A(n+\alpha)^{2}+2 \pi i n \beta\right]=\theta[0, \beta](2 \rho, 0), \\
& C\left(\mathrm{e}_{1}, \mathrm{e}_{1}, \mathrm{e}_{2}\right)=\sum_{n=-\infty}^{\infty} \exp \left[-2 \pi A(n+\alpha)^{2}+2 \pi i(n+1 / 2) \beta\right]=\theta[1 / 2, \beta](2 \rho, 0) .
\end{aligned}
$$

The basis correspondence the involves $\theta[0, \beta](2 \tau, 2 z)$ and $\theta[1 / 2, \beta](2 \tau, 2 z)$. Now it is clear that the translated case with holonomy gives

$$
\begin{aligned}
& C\left(\mathrm{e}_{1}, \mathrm{e}_{1}, \mathrm{e}_{1, \alpha}\right)=\theta[\alpha, \beta](2 \rho, 0), \\
& C\left(\mathrm{e}_{1}, \mathrm{e}_{1}, \mathrm{e}_{2, \alpha}\right)=\theta[1 / 2+\alpha, \beta](2 \rho, 0),
\end{aligned}
$$

and that the $\mathrm{e}_{i, \alpha}$ correspond to $\theta[\alpha, \beta]$ and $\theta[1 / 2+\alpha, \beta]$ (up to phases). But these theta functions are precisely those for the line bundles of degree two described by the point $\alpha \tau+\beta$ on the Jacobian torus. We therefore have learned how the shift functor representing the moduli of different bundles of same rank and degree (i.e. the Jacobian) acts on the Fukaya side by shifting lines, adding holonomy, and introducing phases.

We see that the two real translation parameters describing the Jacobian torus, which are on the same footing from the point of view of line bundles, have different interpretations on the Lagrangian side - in terms of holonomies and displacements of the Lagrangian submanifold. This is perhaps not too surprising if we consider the string theoretic origin of the moduli of the D-brane. Both moduli come from zero modes associated to the 
ten-dimensional gauge field. On the D-brane the transverse components become normal vectors and the zero modes give the motions of the brane, while the parallel components describe the flat bundle moduli (holonomy).

It is now a simple matter to check that these formulas are true for general $\rho$ (recall $\rho=$ $\left.\int_{\widetilde{E}} \omega\right)$, i.e. when $b \neq 0$, since we weight a holomorphic map from the disc by $\exp \left[2 \pi i \int \phi^{*} \omega+\right.$ $\oint \beta d t]$. Complexifying the Kähler class in this way is familiar from mirror symmetry, and the holonomy is expected for D-branes or open strings. We now have a clear description of all the parameters involved in the mirror map $\tau \leftrightarrow \rho$ as well as the translational bundle (or Lagrangian) moduli.

The composition is somewhat more involved for stable vector bundles of rank $r$ and degree $d$ (which correspond to lines $(r, d)$ with $\operatorname{gcd}(r, d)=1$ ), but as we will see in the next section, these cases can be deduced from our knowledge of line bundles. The non-stable bundles (which correspond to non-unitary local systems) are more subtle. Identifying a proper basis for the global sections is the main difficulty. Fortunately, we will be able to describe all sections in terms of simple theta functions. The Fukaya compositions are rather easy to compute, as above, and the equivalence of these products to the decomposition of sections of bundles will be shown to reduce to the classical addition formulas, as above.

\section{Categorical Equivalence for the Elliptic Curve}

We must construct the categorical equivalence in the general case, where the objects in the derived category are more general than line bundles. We can immediately reduce to indecomposable bundles, by linearity of $m_{2}$. The idea, then, is to use the representation of indecomposable vector bundles on elliptic curve as push-forwards under the isogenies of line bundles tensored with bundles of type $F(V, \exp (N))$ where $N$ is a constant nilpotent matrix (see section 2.3). Let us denote by $\mathcal{L}(E)$ the category of bundles on $E$ of the form $L(\phi) \otimes F(V, \exp (N))$. We claim that it is sufficient to construct a natural equivalence of $\mathcal{L}(E)$ with the appropriate subcategory in the Fukaya category. More precisely, we need that these equivalences for elliptic curves $E_{q^{r}}$ and $E_{q}$ commute with the corresponding pull-back functors $\pi_{r}^{*}$.

\section{Main Theorem}

The categories $\mathcal{D}^{b}\left(E_{q}\right)$ and $\mathcal{F}^{0}\left(\widetilde{E}^{q}\right)$ are equivalent:

$$
\mathcal{D}^{b}\left(E_{q}\right) \cong \mathcal{F}^{0}\left(\widetilde{E}^{q}\right)
$$


(we use $q=\exp (2 \pi i \tau)$ instead of $\tau$ here).

We prove this by constructing a bijective functor $\Phi: \mathcal{D}^{b}\left(E_{q}\right) \rightarrow \mathcal{F}^{0}\left(\widetilde{E}^{q}\right)$ in five steps. We first define it on a restricted class of objects: bundles of the form $L(\varphi) \otimes F(V, \exp N)$ (step one). Then we show how this respects the composition law (step two). Step three is showing that it commutes with isogenies. Then, in step four we treat general vector bundles by constructing them from isogenies. Finally, in step five, we treat the only remaining case: torsion sheaves, or thickened skyscrapers (recall that objects in the derived category are, up to shifts, direct sums of vector bundles and these sheaves, which have support only at points).

More specifically, we construct a fully faithful functor from the abelian category of coherent sheaves to the Fukaya category. The choice of logarithms of slopes for the corresponding objects is $\pi i \alpha$, where $\alpha \in(-1 / 2,1 / 2]$ (the right boundary is achieved by torsion sheaves). On this subcategory $\operatorname{Hom}^{0}$ between lines with slopes $\exp \left(\pi i \alpha_{1}\right)$ and $\exp \left(\pi i \alpha_{2}\right)$ is non-zero only if $\alpha_{1}<\alpha_{2}$. This functor can be extended to the equivalence of the entire derived category of coherent sheaves with the Fukaya category, using Serre duality and the rule that the shift by one in the derived category corresponds to $\alpha \mapsto \alpha+1$.

\subsection{The Functor $\Phi$}

Let $\mathcal{L}\left(E_{q}\right)$ denote the full subcategory of the category of vector bundles on $E_{q}$ consisting of bundles of the form $L(\varphi) \otimes F(V, \exp N)$, where $\varphi=t_{x}^{*} \varphi_{0} \cdot \varphi_{0}^{n-1}$ for some $x \in E_{q}, n \in \mathbf{Z}$ (we use the conventions of section 2.3.)

We construct a functor

$$
\Phi_{q}: \mathcal{L}\left(E_{q}\right) \rightarrow \mathcal{F}^{0}\left(\widetilde{E}^{q}\right)
$$

where $\mathcal{F}^{0}$ denotes the Fukaya category and $q$ denotes the exponential of the complexified Kähler parameter on the right hand side $(q=\exp (2 \pi i \rho)$, and $\rho=b+i k$ is set equal to $\tau$, as per the mirror map). On the Fukaya side, an object is a pair of line $\Lambda$ in $\mathbf{R}^{2} / \mathbf{Z}^{2}$ described by a parametrization $(x(t), y(t))$ in $\mathbf{R}^{2}$ and a flat connection $A$ on the line. We describe the flat connection as a constant, $V$-valued one-form on $\mathbf{R}^{2}$ - the restriction to $\Lambda$ is implied. Specifically, the map $\Phi$ on objects is

$$
\begin{aligned}
& \Phi:\left(L\left(t_{\alpha \tau+\beta}^{*} \varphi_{0} \cdot \varphi_{0}^{n-1}\right) \otimes F(V, \exp N)\right) \mapsto(\Lambda, A), \quad \Lambda=(\alpha+t,(n-1) \alpha+n t), \\
& A=\left(-2 \pi i \beta \cdot \mathbf{1}_{V}+N\right) d x
\end{aligned}
$$

(we subsequently drop the notation $\mathbf{1}_{V}$ for the identity map on $V$ ). In other words, the line has slope $n$ (degree of the line bundle) and $x$-intercept $\alpha / n$. The monodromy 
matrix between points $\left(x_{1}, y_{1}\right)$ and $\left(x_{2}, y_{2}\right)$ is $\exp \left[-2 \pi i \beta\left(x_{2}-x_{1}\right)\right] \exp \left[N\left(x_{2}-x_{1}\right)\right]$. This is precislely what we saw last section: the shifts are represented as translations of the line and as monodromies.

It remains to describe the map on morphisms:

$$
\begin{aligned}
\Phi: \operatorname{Hom}\left(L\left(\varphi_{1}\right) \otimes\right. & \left.F\left(V_{1}, \exp N_{1}\right), L\left(\varphi_{2}\right) \otimes F\left(V_{2}, \exp N_{2}\right)\right) \rightarrow \\
& \operatorname{Hom}^{0}\left(\Phi\left(L\left(\varphi_{1}\right) \otimes F\left(V_{1}, \exp N_{1}\right)\right), \Phi\left(L\left(\varphi_{2}\right) \otimes F\left(V_{2}, \exp N_{2}\right)\right)\right),
\end{aligned}
$$

where $\varphi_{i}=t_{\alpha_{i} \tau+\beta_{i}}^{*} \varphi_{0} \cdot \varphi^{n_{i}-1}, i=1,2$, and $\operatorname{Hom}^{0}$ is the image in $\mathcal{F}^{0}$, i.e. the degree zero part of Hom. Now recall Prop. 2. If $n_{1}>n_{2}$, both of the above spaces are zero. If $n_{1}=n_{2}$, then either the above spaces are zero, or $L\left(\varphi_{1}\right) \cong L\left(\varphi_{2}\right)$ and the problem reduces to homomorphisms of vector spaces. If $n_{1}<n_{2}$, then

$$
\begin{aligned}
L H S & =H^{0}\left(L\left(\varphi_{2} \varphi_{1}^{-1}\right) \otimes F\left(V_{1}^{*} \otimes V_{2}, \exp \left(N_{2}-N_{1}^{*}\right)\right)\right) \\
& =H^{0}\left(L\left(\varphi_{2} \varphi_{1}^{-1}\right)\right) \otimes\left(V_{1}^{*} \otimes V_{2}\right) \quad \text { by } \mathcal{V},
\end{aligned}
$$

while

$$
R H S=\bigoplus_{\mathrm{e}_{k} \in \Lambda_{1} \cap \Lambda_{2}} V_{1}^{*} \otimes V_{2} \cdot \mathrm{e}_{k}
$$

The points of $\Lambda_{1} \cap \Lambda_{2}$ are easily found from $\Phi$ to be

$$
\mathrm{e}_{k}=\left(\frac{k+\alpha_{2}-\alpha_{1}}{n_{2}-n_{1}}, \frac{n_{1} k+n_{1} \alpha_{2}-n_{2} \alpha_{1}}{n_{2}-n_{1}}\right), \quad k \in \mathbf{Z} /\left(n_{2}-n_{1}\right) \mathbf{Z} .
$$

We note that

$$
\varphi_{2} \varphi_{1}^{-1}=t_{\alpha_{2} \tau+\beta_{2}}^{*} \varphi_{0} \cdot \varphi_{0}^{n_{2}-1} \cdot t_{\alpha_{1} \tau+\beta_{1}}^{*} \varphi_{0}^{-1} \varphi_{0}^{-n_{1}+1}=t_{\alpha_{12} \tau+\beta_{12}}^{*} \varphi_{0}^{n_{2}-n_{1}}
$$

where

$$
\alpha_{12}=\frac{\alpha_{2}-\alpha_{1}}{n_{2}-n_{1}}, \quad \beta_{12}=\frac{\beta_{2}-\beta_{1}}{n_{2}-n_{1}} .
$$

Now we have the standard basis of theta functions on $H^{0}\left(L\left(\varphi_{2} \varphi_{1}^{-1}\right)\right)$ :

$$
\begin{aligned}
& t_{\alpha_{12} \tau+\beta_{12}}^{*} \theta\left[\frac{k}{n_{2}-n_{1}}, 0\right]\left(\left(n_{2}-n_{1}\right) \tau,\left(n_{2}-n_{1}\right) z\right)= \\
& \theta\left[\frac{k}{n_{2}-n_{1}}, 0\right]\left(\left(n_{2}-n_{1}\right) \tau,\left(n_{2}-n_{1}\right)\left(z+\alpha_{12} \tau+\beta_{12}\right)\right),
\end{aligned}
$$

$k \in \mathbf{Z} /\left(n_{2}-n_{1}\right) \mathbf{Z}$. Let us call this function $f_{k}$. The standard basis on the Fukaya side is also indexed by $k$. We recall that $\alpha_{12}$ and $\beta_{12}$ effect shifts and monodromies on the right hand 
side, and this determines the identification of bases up to a constant. Let $T \in V_{1}^{*} \otimes V_{2}$. We define

$\Phi\left(\mathcal{V}\left(f_{k} \otimes T\right)\right)=\exp \left(-\pi i \tau \alpha_{12}^{2}\left(n_{2}-n_{1}\right)\right) \exp \left[\alpha_{12}\left(N_{2}-N_{1}^{*}-2 \pi i\left(n_{2}-n_{1}\right) \beta_{12}\right)\right] \cdot T \mathrm{e}_{k}$

\section{2. $\Phi \circ m_{2}=m_{2} \circ \Phi$}

We must check that the definition of $\Phi$ respects the composition maps in the two categories. We have $L\left(\varphi_{i}\right) \otimes F\left(V_{i}, \exp N_{i}\right), i=1 \ldots 3$.

a) We have to compare compositions of sections

$$
\mathcal{V}\left(t_{\alpha_{12} \tau+\beta_{12}}^{*} \theta\left[\frac{a}{n_{2}-n_{1}}, 0\right]\left(\left(n_{2}-n_{1}\right) \tau,\left(n_{2}-n_{1}\right) z\right) \otimes A\right)
$$

and

$$
\mathcal{V}\left(t_{\alpha_{23} \tau+\beta_{23}}^{*} \theta\left[\frac{a}{n_{3}-n_{2}}, 0\right]\left(\left(n_{3}-n_{2}\right) \tau,\left(n_{3}-n_{2}\right) z\right) \otimes B\right),
$$

where $A \in V_{1}^{*} \otimes V_{2}$ and $B \in V_{2}^{*} \otimes V_{3}$. Using Prop. 3, this composition is equal to $\mathcal{V}$ applied to the following expression:

$$
\begin{aligned}
\operatorname{Tr}_{V_{2}} \theta\left[\frac{a}{n_{2}-n_{1}}, 0\right]\left(\left(n_{2}-n_{1}\right) \tau,\left(n_{2}-n_{1}\right)\left(z+\alpha_{12} \tau+\beta_{12}-\frac{N}{n_{2}-n_{1}}\right)\right) \times \\
\quad \times \theta\left[\frac{b}{n_{3}-n_{2}}, 0\right]\left(\left(n_{3}-n_{2}\right) \tau,\left(n_{3}-n_{2}\right)\left(z+\alpha_{23} \tau+\beta_{23}-\frac{N}{n_{3}-n_{2}}\right)\right) \cdot A \otimes B
\end{aligned}
$$

where

$$
\begin{aligned}
N & =\frac{1}{2 \pi i} \frac{-\left(n_{2}-n_{1}\right)\left(N_{3}-N_{2}\right)+\left(n_{3}-n_{2}\right)\left(N_{2}-N_{1}^{*}\right)}{n_{3}-n_{1}} \\
& =\frac{1}{2 \pi i}\left(N_{2}-\frac{\left(n_{2}-n_{1}\right) N_{3}+\left(n_{3}-n_{2}\right) N_{1}^{*}}{n_{3}-n_{1}}\right)
\end{aligned}
$$

(we have replaced $N_{2}^{*}$ by $N_{2}$ under the trace sign using $\left\langle v, N_{2}^{*} \xi\right\rangle=\left\langle N_{2} v, \xi\right\rangle$ for $v \in V_{2}, \xi \in$ $V_{2}^{*}$ ). Now the addition formula (II.6.4 of [19]) implies the following $\theta$-identity:

$$
\begin{array}{r}
\theta\left[\frac{a}{n_{2}-n_{1}}, 0\right] \\
=\sum_{m \in \mathbf{Z}} \exp \left[\pi i \tau \frac{b}{\left(n_{2}-n_{1}\right)\left(n_{3}-n_{2}\right)\left(n_{3}-n_{1}\right)}+2 \pi i \frac{k_{m}}{n_{3}-n_{1}}\left(z_{2}-z_{1}\right)\right] \times \\
\left.\theta\left[\frac{a+b+\left(n_{3}-n_{2}\right) m}{n_{3}-n_{1}}, 0\right]\left(\left(n_{3}-n_{1}\right) \tau,\left(n_{2}-n_{1}\right) z_{1}+\left(n_{3}-n_{2}\right)\right) \cdot\left(n_{2}\right) z_{2}\right),
\end{array}
$$

where

$$
k_{m}=\left(n_{2}-n_{1}\right) b-\left(n_{3}-n_{2}\right) a+\left(n_{2}-n_{1}\right)\left(n_{3}-n_{2}\right) m
$$


Plugging in $z_{1}=z+\alpha_{12} \tau+\beta_{12}-\frac{N}{n_{2}-n_{1}}$ and $z_{2}=z+\alpha_{12} \tau+\beta_{12}-\frac{N}{n_{2}-n_{1}}$, we obtain

$$
\begin{aligned}
\operatorname{Tr}_{V_{2}} \sum_{m \in \mathbf{Z}} \exp [ & \frac{(\pi i \tau) k_{m}^{2}}{\left(n_{2}-n_{1}\right)\left(n_{3}-n_{2}\right)\left(n_{3}-n_{1}\right)} \\
& \left.+\frac{(2 \pi i) k_{m}}{n_{3}-n_{1}}\left(\left(\alpha_{23}-\alpha_{12}\right) \tau+\beta_{23}-\beta_{12}+\frac{n_{3}-n_{1}}{\left(n_{2}-n_{1}\right)\left(n_{3}-n_{2}\right)} N\right)\right] \cdot A \otimes B \times \\
& \times \theta\left[\frac{a+b+\left(n_{3}-n_{2}\right) m}{n_{3}-n_{1}}, 0\right]\left(\left(n_{3}-n_{1}\right)\left(z+\alpha_{13} \tau+\beta_{13}\right)\right) .
\end{aligned}
$$

b) In the Fukaya category, we need to compose $A \cdot \mathrm{e}_{a}$ and $B \cdot \mathrm{e}_{b}$, where $A \in V_{1}^{*} \otimes V_{2}$ and $B \in V_{2}^{*} \otimes V_{3}$ and $a \in \mathbf{Z} /\left(n_{2}-n_{1}\right) \mathbf{Z}$ and $b \in \mathbf{Z} /\left(n_{3}-n_{2}\right) \mathbf{Z}$ label points in $\Lambda_{1} \cap \Lambda_{2}$ and $\Lambda_{2} \cap \Lambda_{3}$, respectively.

The points of intersection are easily computed. For two lines $\Lambda_{i}$ and $\Lambda_{j}$, the $n_{j}-n_{i}$ points of intersection in $\mathbf{R}^{2} / \mathbf{Z}^{2}$ are

$$
\mathrm{e}_{k}=\left(\alpha_{i j}+\frac{k}{n_{j}-n_{i}}, \frac{n_{i} \alpha_{j}-n_{j} \alpha_{i}+n_{i} k}{n_{j}-n_{i}}\right) .
$$

We must sum over triangles in the plane formed by the $\mathbf{Z}$-translates of $\Lambda_{3}$ which pass through points lattice-equivalent to $\mathrm{e}_{b}$. Note that such a triangle is uniquely determined by the first coordinate of the intersection point of $\Lambda_{3}$ with $\Lambda_{2}$ which should be of the form $\alpha_{23}+\frac{b}{n_{3}-n_{2}}+m, m \in \mathbf{Z}$. The point of intersection $\mathrm{e}_{c}$ in $\Lambda_{1} \cap \Lambda_{3}$ depends on $m$, and is calculated to be given by the above formula with $c=a+b+m\left(n_{3}-n_{2}\right) \bmod \left(n_{3}-n_{1}\right)$.

If we call $l_{1}$ the horizontal distance (difference of $x$ coordinates) between $\mathrm{e}_{a}$ and $\mathrm{e}_{c}, l_{2}$ the horizontal distance between $\mathrm{e}_{a}$ and $\mathrm{e}_{b}$, and $l_{3}$ the horizontal distance between $\mathrm{e}_{b}$ and $\mathrm{e}_{c}$ (may be negative), then by adding up $x$ and $y$ differences we have

$$
\begin{aligned}
l_{1} & =l_{2}+l_{3} \\
n_{1} l_{1} & =n_{2} l_{2}+n_{3} l_{3} .
\end{aligned} .
$$

Therefore, $l_{1}=\frac{n_{3}-n_{2}}{n_{3}-n_{1}} l_{2}$, and $l_{3}=\frac{n_{1}-n_{2}}{n_{3}-n_{1}} l_{2}$. Now we know $l_{2}$ explicitly from above. The area $\Delta$ of the triangle (rather, the number of fundamental domains) formed by the vertices is half that of the parallelogram determined by vectors $\left(l_{1}, n_{1} l_{1}\right)$ and $\left(l_{2}, n_{2} l_{2}\right)$. Hence,

$$
\Delta=\frac{1}{2} \operatorname{Det}\left(\begin{array}{cc}
l_{1} & l_{2} \\
n_{1} l_{1} & n_{2} l_{2}
\end{array}\right)=l_{1} l_{2}\left(n_{2}-n_{1}\right) / 2=\frac{\left(n_{3}-n_{1}\right)\left(n_{2}-n_{1}\right)}{2\left(n_{3}-n_{2}\right)}\left(l_{2}\right)^{2} .
$$

7 There is some notational ambiguity: "What is $e_{3}$ ?" The context should make it clear which intersections are being indexed, and therefore what the range of the index should be. 
Plugging in $l_{2}=\alpha_{23}-\alpha_{12}+b /\left(n_{3}-n_{2}\right)-a /\left(n_{2}-n_{1}\right)+m$, gives

$$
\Delta=\frac{1}{2} \frac{\left[k_{m}+\left(\alpha_{23}-\alpha_{12}\right)\left(n_{3}-n_{2}\right)\left(n_{2}-n_{1}\right)\right]^{2}}{\left(n_{3}-n_{2}\right)\left(n_{3}-n_{1}\right)\left(n_{2}-n_{1}\right)}
$$

where $k_{m}$ was defined above. Now since $\int \phi^{*} \omega=\rho \Delta=\tau \Delta$, then recalling the assigned monodromies, we have, by (3.1) for the Fukaya product (recalling the sign conventions of the $l_{i}$ ),

$$
\begin{gathered}
A \cdot \mathrm{e}_{a} \circ B \cdot \mathrm{e}_{b}= \\
\sum_{m \in \mathbf{Z}} \exp \left\{\pi i \tau \frac{\left[k_{m}+\left(\alpha_{23}-\alpha_{12}\right)\left(n_{3}-n_{2}\right)\left(n_{2}-n_{1}\right)\right]^{2}}{\left(n_{3}-n_{2}\right)\left(n_{3}-n_{1}\right)\left(n_{2}-n_{1}\right)}\right\} \times \\
\operatorname{Tr}_{V_{2}} \exp \left[-l_{1}\left(N_{1}^{*}-2 \pi i \beta_{1}\right)+l_{2}\left(N_{2}-2 \pi i \beta_{2}\right)+l_{3}\left(N_{3}-2 \pi i \beta_{3}\right)\right] . \\
\cdot A \otimes B \cdot \mathrm{e}_{a+b+m\left(n_{3}-n_{2}\right)} .
\end{gathered}
$$

Upon expanding the square, substituting the correct values of the $l_{i}$, and recalling the definition of $N$, the right hand side becomes

$$
\begin{aligned}
\operatorname{Tr}_{V_{2}} \sum_{m \in \mathbf{Z}} \exp [ & \pi i \tau \frac{k_{m}^{2}}{\left(n_{3}-n_{2}\right)\left(n_{3}-n_{1}\right)\left(n_{2}-n_{1}\right)}+2 \pi i \frac{k_{m}}{n_{3}-n_{1}}\left(\left(\alpha_{23}-\alpha_{12}\right) \tau+\beta_{23}-\beta_{12}\right) \\
& +\pi i \tau\left(\alpha_{23}-\alpha_{12}\right)^{2}\left(n_{3}-n_{2}\right) \frac{n_{2}-n_{1}}{n_{3}-n_{1}} \\
& +2 \pi i\left(\alpha_{23}-\alpha_{12}\right)\left(\beta_{23}-\beta_{12}\right) \frac{\left(n_{3}-n_{2}\right)\left(n_{2}-n_{1}\right)}{n_{3}-n_{1}} \\
& \left.+\frac{k_{m}+\left(\alpha_{23}-\alpha_{12}\right)\left(n_{3}-n_{2}\right)\left(n_{2}-n_{1}\right)}{\left(n_{3}-n_{2}\right)\left(n_{2}-n_{1}\right)} 2 \pi i N\right] \cdot A \otimes B \cdot \mathrm{e}_{a+b+m\left(n_{3}-n_{2}\right)} .
\end{aligned}
$$

Taking into account the exponential factors in the definition of $\Phi$ yields equivalence with the product in the derived category, which is the desired result.

\subsection{Isogeny}

On our subcategory $\mathcal{L}$, we have the functor of pull-back under isogeny:

$$
\pi_{r}^{*}: \mathcal{L}\left(E_{q}\right) \rightarrow \mathcal{L}\left(E_{q^{r}}\right)
$$

We describe the analogue on the Fukaya side. Consider $\pi_{r}: \mathbf{R}^{2} / \mathbf{Z}^{2} \rightarrow \mathbf{R}^{2} / \mathbf{Z}^{2}$, which sends $(x, y)$ to $(r x, y)$. This is an $r$-fold covering and respects the Kähler form if the complexified Kähler parameter on the left hand side is $r \rho$ when that of the right hand side is $\rho$. We then have the corresponding functors $\pi_{r}^{*}$ and $\pi_{r *}$ between Fukaya categories. 


\section{Proposition 4}

One has the following commutative diagram of functors:

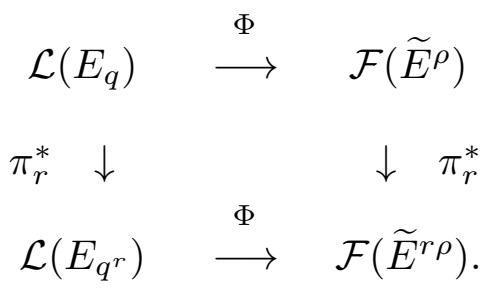

Proof: This is a straightforward check using the formula

$$
\theta[a / n, 0](n \tau, n z)=\sum_{k \in \mathbf{Z} / r \mathbf{Z}} \theta[(a+n k) /(n r), 0]\left(n r^{2} \tau, n r z\right)
$$

\subsection{The General Case}

Now we can extend the functor $\Phi$ from the category $\mathcal{L}\left(E_{q}\right)$ to the category of all vector bundles. For this we use the fact that any indecomposable bundle on $E_{q}$ is isomorphic to

$$
\pi_{r *}\left(L_{q^{r}}(\varphi) \otimes F_{q^{r}}(V, \exp N)\right)
$$

(see Prop. 1). Hence we can set

$$
\Phi_{q}\left(\pi_{r *}\left[L_{q^{r}}(\varphi) \otimes F_{q^{r}}(V, \exp N)\right]\right)=\pi_{r *}\left(\Phi_{q^{r}}\left[L_{q^{r}}(\varphi) \otimes F_{q^{r}}(V, \exp N)\right]\right)
$$

It remains to define $\Phi_{q}$ on morphisms between vector bundles. For this we use the isomorphism (2.2):

$$
\begin{array}{r}
\operatorname{Hom}\left(\pi_{r_{1} *}\left[L_{q^{r_{1}}}(\varphi) \otimes F_{q^{r_{1}}}\left(V_{1}, \exp N_{1}\right)\right], \pi_{r_{2} *}\left[L_{q^{r_{2}}}(\varphi) \otimes F_{q^{r_{2}}}\left(V_{2}, \exp N_{2}\right)\right]\right) \cong \\
\operatorname{Hom}\left(\pi_{2}^{*}\left[L_{q^{r_{1}}}(\varphi) \otimes F_{q^{r_{1}}}\left(V_{1}, \exp N_{1}\right)\right], \pi_{1}^{*}\left[L_{q^{r_{2}}}(\varphi) \otimes F_{q^{r_{2}}}\left(V_{2}, \exp N_{2}\right)\right]\right),
\end{array}
$$

where $\pi_{1}$ and $\pi_{2}$ are defined from the cartesian square

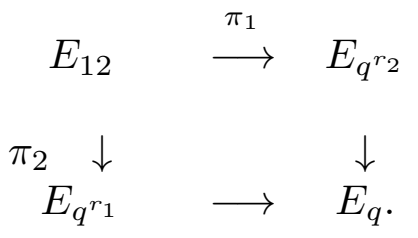

Here $E_{12}$ is an elliptic curve if and only if $\operatorname{gcd}\left(r_{1}, r_{2}\right)=1$. In general, $E_{12} \cong E_{q_{1}^{r_{1}^{\prime} r_{2}^{\prime}}} \times \mathbf{Z} / d \mathbf{Z}$, where $d=\operatorname{gcd}\left(r_{1}, r_{2}\right)$ and $r_{i}^{\prime}=r_{i} / d, i=1,2$. Thus, the above Hom space is decomposed 
into a direct sum of $d$ Hom spaces between objects of the category $\mathcal{L}\left(E_{q_{1} r_{2}^{\prime}}\right)$. There is a similar decomposition of the corresponding Hom spaces on the Fukaya side, so we just take the direct sum of isomorphisms between Hom's given by $\Phi$ on $\mathcal{L}\left(E_{q_{1}^{r_{1}^{\prime}} r_{2}^{\prime}}\right)$. To check that this extended map $\Phi$ is still compatible with compositions we note that given a triple of vector bundles of the form $\pi_{r_{i} *}\left(L_{q^{r_{i}}}\left(\varphi_{i}\right) \otimes F_{q^{r_{i}}}\left(V_{i}, \exp N_{i}\right)\right), i=1 \ldots 3$, we can embed the pairwise Hom's into the corresponding Hom spaces between the pull-backs of $L_{q^{r_{i}}}\left(\varphi_{i}\right) \otimes F_{q^{r_{i}}}\left(V_{i}, \exp N_{i}\right)$ to the triple fibered product of $E_{q^{r_{1}}}, E_{q^{r_{2}}}$, and $E_{q^{r_{3}}}$ over $E_{q}$, which is still a disjoint union of elliptic curves. Now the required compatibility follows from the fact that the functor $\Phi$ on the $\mathcal{L}\left(E_{q}\right)$ commutes with the pull-back functor $\pi_{r}^{*}$.

\subsection{Extension to Torsion Sheaves}

For every $z_{0} \in \mathbf{C}$ and a nilpotent operator $N \in \operatorname{End}(V)$ we have the corresponding coherent sheaf of $\mathbf{C}$ supported at $z_{0}$. Namely, $\mathcal{O}_{r z_{0}} \otimes V /\left\langle z-z_{0}-\frac{N}{2 \pi i}\right\rangle$, where $r=\operatorname{dim} V$ is the smallest positive integer such that $N^{r}=0$. For example, if $N=0$ then the notation means that we set to zero anything in the ideal generated by $z-z_{0}$, i.e. we get $\mathcal{O}_{z_{0}}$ as in (2.1). We denote by $S\left(z_{0}, V, N\right)$ the direct image of this sheaf on $E$. Then for every object $L(\varphi) \otimes F\left(V^{\prime}, \exp N^{\prime}\right)$ of $\mathcal{L}(E)$ we have canonically $\operatorname{Hom}\left(L(\varphi) \otimes F\left(V^{\prime}, \exp N^{\prime}\right), S\left(z_{0}, V, N\right)\right) \cong \operatorname{Hom}\left(V^{\prime}, V\right)$. The composition map

$$
\begin{gathered}
\operatorname{Hom}\left(L\left(\varphi_{1}\right) \otimes F\left(V_{1}, \exp N_{1}\right), L\left(\varphi_{2}\right) \otimes F\left(V_{2}, \exp N_{2}\right)\right) \otimes \\
\operatorname{Hom}\left(L\left(\varphi_{2}\right) \otimes F\left(V_{2}, \exp N_{2}\right), S\left(x, V_{3}, N_{3}\right)\right) \longrightarrow \\
\operatorname{Hom}\left(L\left(\varphi_{1}\right) \otimes F\left(V_{1}, \exp N_{1}\right), S\left(x, V_{3}, N_{3}\right)\right)
\end{gathered}
$$

can be written (using the isomorphism $\mathcal{V}$ ) as follows:

$$
\mathcal{V}(f \otimes A) \circ B=\operatorname{Tr}_{V_{2}} f\left(x+\frac{N_{3}}{2 \pi i}-\frac{1}{2 \pi i\left(n_{2}-n_{1}\right)}\left(N_{2}-N_{1}\right)^{*}\right)(A \otimes B),
$$

where $f \in H^{0}\left(L\left(\varphi_{2} \varphi_{1}^{-1}\right)\right), A \in \operatorname{Hom}\left(V_{1}, V_{2}\right)$, and $B \in \operatorname{Hom}\left(V_{2}, V_{3}\right)$.

We extend $\Phi$ to torsion sheaves by sending $S(\alpha \tau+\beta, V, N)$ to the line $(-\alpha, t)$ with connection given by $2 \pi i \beta d y$. We also define an isomorphism

$$
\begin{aligned}
\Phi: \operatorname{Hom}\left(L\left(\varphi_{1}\right) \otimes F\left(V_{1}, \exp N_{1}\right), S\left(x, V_{2}, N_{2}\right)\right) \longrightarrow \\
\operatorname{Hom}\left(\Phi\left(L\left(\varphi_{1}\right) \otimes F\left(V_{1}, \exp N_{1}\right)\right), \Phi\left(S\left(x, V_{2}, N_{2}\right)\right)\right) \cong \operatorname{Hom}\left(V_{1}, V_{2}\right)
\end{aligned}
$$

by the formula (where $\varphi_{1}=t_{\alpha_{1} \tau+\beta_{1}}^{*} \varphi_{0} \cdot \varphi_{0}^{n-1}$ and $x=\alpha_{2} \tau+\beta_{2}$ )

$$
A \mapsto \exp \left[-\pi i \tau n \alpha_{2}^{2}-2 \pi i \alpha_{1} \alpha_{2}-\alpha_{2}\left(n N_{2}-N_{1}^{*}+2 \pi i\left(\beta_{1}+n \beta_{2}\right)\right)-\alpha_{1} N_{2}\right] \cdot A,
$$


where $A \in \operatorname{Hom}\left(V_{1}, V_{2}\right)$.

It remains to check that $\Phi$ respects compositions. We only have to check this for the composition of Hom's between $L\left(\varphi_{1}\right) \otimes F\left(V_{1}, \exp N_{1}\right), L\left(\varphi_{2}\right) \otimes F\left(V_{2}, \exp N_{2}\right)$, and $S\left(x, V_{3}, N_{3}\right)$. The proof of this is similar to the cases considered previously, but even simpler. One needs only the definition of the theta function; no theta identities are used. We leave the case of Hom's from higher rank vector bundles as an exercise for the reader (use $\pi_{r *}$ ).

\section{Remark}

Any automorphism $\varphi$ of $\widetilde{M}$ preserving the Kähler structure and $\Omega$ induces an autoequivalence $\varphi_{*}$ of the Fukaya category $\mathcal{F}(\widetilde{\mathcal{M}})$ defined up to a shift by an integer. In the case of the flat torus $T=\mathbf{R}^{2} / \mathbf{Z}^{2}$, this leads to an action of the central extension of $S L(2 ; \mathbf{Z})$ by $\mathbf{Z}$ in the Fukaya category $\mathcal{F}(T)$. The corresponding projective action of $S L(2 ; \mathbf{Z})$ on $\mathcal{D}^{b}(E)$ is given as follows. The matrix $\left(\begin{array}{ll}1 & 0 \\ 1 & 1\end{array}\right)$ acts by tensoring with $L$, while the matrix $\left(\begin{array}{cc}0 & 1 \\ -1 & 0\end{array}\right)$ acts as the Fourier-Mukai transform

$$
\mathcal{S} \mapsto p_{2 *}\left(p_{1}^{*} \mathcal{S} \otimes \mathcal{P}\right)
$$

where $\mathcal{P}$ is the normalized Poincaré line bundle on $E \times E$ given by

$$
\mathcal{P}=m^{*} \mathcal{O}\left(-x_{0}\right) \otimes p_{1}^{*} \mathcal{O}\left(x_{0}\right) \otimes p_{2}^{*} \mathcal{O}\left(x_{0}\right)
$$

where $m: E \times E \rightarrow E$ is the group law, $x_{0}$ is the point corresponding to $1 / 2+\tau / 2$, and $p_{1}, p_{2}$ are the projections.

\section{Conclusions}

We have shown that for the elliptic curve, as Kontsevich conjectured, mirror symmetry has an interpretation in terms of the equivalence of two very different looking categories. The nature of the equivalence certainly seems to equate odd D-branes (the Fukaya side) with even D-branes, though the interpretation of D-branes as coherent sheaves (much less a elements of the derived category) has as yet only been speculative [20], [21].

We have offered a detailed proof of categorical mirror symmetry in the case of the elliptic curve. The composition law contains a wealth of information about holomorphic discs in the mirror manifold, though it is not yet clear exactly how the many results of 
ordinary mirror symmetry would follow from categorical mirror symmetry for a general Calabi-Yau manifold.

We have given the dictionary for the categories, but it has not been through a construction. The lack of a constructive approach not only hinders attempts towards a general proof but makes it difficult to even guess at the dictionary between objects for other Calabi-Yau mirror pairs. One wonders: Is it at all possible to construct the sheaf on $M$ corresponding to a given special-Lagrangian submanifold (and local system) on $\widetilde{M}$ ? For $E$ and $\widetilde{E}$ we can answer in the affirmative with the following construction. 8

Let $\Lambda=(r, d)$ be a line on $\widetilde{E}^{\tau}=\mathbf{R}^{2} / \mathbf{Z}^{2}$. Let $\Lambda_{1}$ denote the diagonal line $(1,1)$. Then $\Lambda_{1} \times \Lambda \subset \widetilde{E} \times \widetilde{E}$ can be parametrized by $(s, t) \in S^{1} \times S^{1}$ by

$$
(s, t) \mapsto(s, s, r t, d t)
$$

Anticipating the isogeny argument, we can put a complex structure on $\Lambda_{1} \times \Lambda$ identifying it with $E_{r \tau}$, i.e. we choose the complex coordinate $v=s+\operatorname{tr} \tau$. Then we choose a complex structure on $\widetilde{E} \times \widetilde{E}$ such that $\Lambda_{1} \times \Lambda \cong E_{r \tau}$ is a complex submanifold. The choice is essentially unique: we take

$$
\begin{aligned}
& w_{1}=x_{1}+x_{3} \tau \\
& w_{2}=x_{2}+\frac{r}{d} x_{4} \tau .
\end{aligned}
$$

The submanifold $\Lambda_{1} \times \Lambda$ is then represented as an imbedding

$$
\iota: E_{r \tau} \rightarrow E_{\tau} \times E_{(r / d) \tau}
$$

simply by $v \mapsto(v, v)$. On $E_{(r / d) \tau}$ lives the line bundle $L$ of degree one (whose section is $\theta((r / d) \tau, z))$, and by pulling back $L$ under the projection $p_{2}$ to the second factor, we get $D \equiv p_{2}^{*} L$, a line bundle on $E_{\tau} \times E_{(r / d) \tau}$. We form the appropriate vector bundle over $E_{\tau}$ by taking $\pi_{r *} \iota^{*} D$. One checks that this coincides with our construction of the vector bundle over $E_{\tau}$ associated to the trivial local system on $\Lambda$. Note that if we let $\mathbf{R} / \mathbf{Z}$ act on $\widetilde{E}$ by $t \cdot(x, y)=(t+x, y)$ then $D$ is almost uniquely defined as a $\mathbf{R} / \mathbf{Z} \times \mathbf{R} / \mathbf{Z}$-equivariant holomorphic line bundle on $\widetilde{E} \times \widetilde{E}$ with one-dimensional space of holomorphic sections. More precisely, if in addition we require that $D$ is invariant under the inversion map $z \mapsto-z$, and all its holomorphic sections are even, then there are three such line bundles, one of them is $p_{2}^{*} L$. Thus, the construction is almost canonical once we fix the above $\mathbf{R} / \mathbf{Z}$-action on $\widetilde{E}$ and the $\mathbf{R} / \mathbf{Z}$-equivariant projection $\widetilde{E} \rightarrow \mathbf{R} / \mathbf{Z}:(x, y) \mapsto x$.

8 We thank S.-T. Yau for a conversation on this issue. 
One cannot at present be too optimistic about generalizing this result to higherdimensional Calabi-Yau manifolds. The problem is that the Fukaya category composition depends on the Kähler form, i.e. on the unique Calabi-Yau metric. The exact form of this metric is of course not known. Abelian surfaces offer the only possible simple extensions of this work - this is work in progress. Some hope may lie in the fact that areas of calibrated submanifolds are topoligical, in a way, as the volume is the restriction of a closed form.

Another obstacle is that the derived category of coherent sheaves on a general CalabiYau manifold is not easily studied. For K3 some facts are known [13], and through the work of [22] we can expect some simplification for elliptically fibered manifolds, but a precise statement of the composition eludes us. One may hope to construct a map between objects, however. This may be a possible starting point. For example, the conjecture of [7] can be interpreted as simply positing an object on the Fukaya side equivalent to the skyscraper sheaf over a point on the mirror (derived category) side - the identification of the moduli space of this object is immediately seen to be the mirror manifold itself. Though Lagrangian submanifolds with flat $U(1)$ bundles (the conjectured mirror objects to the skyscrapers) may not seem too natural, we recall that the prequantum line bundle, whose first Chern class equals the Kähler class, restricts to a flat line bundle on Lagrangian submanifolds in the Calabi-Yau.

Even less is known about the Fukaya category. Special-Lagrangian submanifolds are not a well-studied class of objects. Recent work of Hitchin [23] has only begun the investigation of these spaces as dually equivalent to complex submanifolds.

Finally, we anticipate a return to the connection of [24], in which the derived category of coherent sheaves on a Fano variety was used to count the number of solitions of a supersymmetric sigma model on that manifold. In that context, the moduli space was the space of topological field theories, or in other words the "enlarged" moduli space. Armed with the interpretation of D-branes as solitonic boundary states in a superconformal field theory, we learn through Kontsevich's work that the appearance of the derived category, while still somewhat mysterious, is not likely to be accidental.

We look forward to further research on these matters.

\section{Acknowledgements}

The research of A.P. is supported in part by NSF grant DMS-9700458, and that of E.Z. by grant DE-F602-88ER-25065. 


\section{References}

[1] B. R. Greene and M. R. Plesser, "Duality in Calabi-Yau Moduli Space," Nucl. Phys. B338 (1990) 15-37.

[2] P. Candelas, X. C. De La Ossa, P. Green, and L Parkes, "A Pair of Calabi-Yau Manifolds as an Exactly Soluble Superconformal Theory," Nucl. Phys. B359 (1991) 21.

[3] M. Kontsevich, "Homological Algebra of Mirror Symmetry," Proceedings of the 1994 International Congress of Mathematicians I, Birkäuser, Zürich, 1995, p. 120; alggeom/9411018.

[4] Y. Ruan and G. Tian, A Mathematical Theory of Quantum Cohomology," J. Diff. Geom. 42 (1995) 259-367.

[5] E. Witten, "Mirror Manifolds and Topological Field Theory," in Essays on Mirror Symmetry, S.-T. Yau, ed., International Press, Hong Kong, 1992, pp. 120-159.

[6] M. Kontsevich, "Deformation Quantization of Poisson Manifolds, I," q-alg/9709040; S. Barannikov and M. Kontsevich, "Frobenius Manifolds and Formality of Lie Algebras of Polyvector Fields," alg-geom/9710032.

[7] A. Strominger, S.-T. Yau, and E. Zaslow, "Mirror Symmetry is T-Duality," Nucl. Phys. B479 (1996) 243-259.

[8] C. Vafa and E. Witten, "On Orbifolds with Discrete Torsion," J. Geom. Phys. 15 (1995) 189.

[9] B.Acharya, "A Mirror Pair of Calabi-Yau Fourfolds in Type II String Theory," hepth/9703029.

[10] M. Gross and P. M. H. Wilson, "Mirror Symmetry via 3-Tori for a Class of Calabi-Yau Threefolds," alg-geom/9608004.

[11] N. C. Leung and C. Vafa, "Branes and Toric Geometry," hep-th/9711013.

[12] K. Fukaya, Morse Homotopy, $A^{\infty}$-Category, and Floer Homologies," in The Proceedings of the 1993 GARC Workshop on Geometry and Topology, H. J. Kim, ed., Seoul National University; "Floer Homology, $A^{\infty}$-Categories and Topological Field Theory" (notes by P. Seidel), Kyoto University preprint Kyoto-Math 96-2.

[13] D. Orlov, "Equivalences of Derived Categories and K3 Surfaces," alg-geom/9606006.

[14] P. Griffiths and J. Harris, Principles of Algebraic Geometry, Wiley \& Sons, New York, 1978.

[15] M. F. Atiyah, "Vector Bundles over an Elliptic Curve," Proc. Lond. Math. Soc. VII (1957) 414-452.

[16] R. Harvey and H. B. Lawson, Jr., "Calibrated Geomtries," Acta Math. 148 (1982) $47-157$.

[17] K. Becker, M. Becker, and A. Strominger, "Fivebranes, Membranes, and NonPerturbative String Theory," hep-th/9507158. 
[18] J. Stasheff, "Homotopy Associativity of $H$-Spaces. I and II," Trans. Amer. Math. Soc 108 (1963) 275-292 and 293-312.

[19] D. Mumford, Tata Lectures on Theta I, Birkhäuser, Boston, 1983.

[20] J. A. Harvey and G. Moore, "On the Algebras of BPS States," hep-th/9609017.

[21] D. R. Morrison, "The Geometry Underlying Mirror Symmetry," alg-geom/9608006.

[22] R. Friedman, J. Morgan, and E. Witten, "Vector Bundles and F Theory," hepth/9701162.

[23] N. Hitchin, "The Moduli Space of Special Lagrangian Submanifolds," dg-ga/9711002.

[24] E. Zaslow, "Solitons and Helices: The Search for a Math-Physics Bridge," Commun. Math. Phys. 175 (1996) 337-375. 